\title{
Oral contraception and eye disease: findings in two large cohort studies
}

\author{
M P Vessey, P Hannaford, J Mant, R Painter, P Frith, D Chappel
}

\begin{abstract}
Aim-To investigate the relation between oral contraceptive use and certain eye diseases.

Methods-Abstraction of the relevant data from the two large British cohort studies of the effects of oral contraception, the Royal College of General Practitioners' (RCGP) Oral Contraception Study and the Oxford-Family Planning Association (Oxford-FPA) Contraceptive Study. Both cohort studies commenced in 1968 and were organised on a national basis. $\mathrm{Be}$ tween them they have accumulated over 850000 person years of observation involving 63000 women.

Results-The conditions considered in the analysis were conjunctivitis, keratitis, iritis, lacrimal disease, strabismus, cataract, glaucoma, retinal detachment, and retinal vascular lesions. With the exception of retinal vascular lesions, there was no consistent evidence of important increases in risk of eye diseases in users of oral contraception. There was about a twofold increase in the risk of retinal vascular lesions in recent pill users in both studies (statistically significant only in the RCGP study). The increase was not limited to any specific type of lesion and may well reflect diagnostic bias.

Conclusion-Oral contraceptive use does not appear to increase the risk of eye disease, with the possible exception of retinal vascular lesions.

(Br f Ophthalmol 1998;82:538-542)
\end{abstract}

J Mant

R Painter

Department of

General Practice and

Primary Care,

Foresterhill Health

Centre, Aberdeen

AB25 2AY

P Hannaford

Oxford Eye Hospital, Radcliffe Infirmary, Oxford OX2 6HE

P Frith

Department of Epidemiology and Public Health, The Medical School,

Newcastle on Tyne

NE2 4HH

D Chappel

Correspondence to: Professor M P Vessey.

Accepted for publication 27 November 1997
Numerous case reports were published during the 1960s and 1970s concerning the occurrence of a variety of eye disorders in women using oral contraceptives. ${ }^{1}$ Epidemiological studies at that time were few and too small to provide clear results. More recently, a casecontrol study has found no association between use of oral contraception and early with insulin dependent diabetes. ${ }^{2}$ The Beaver Dam survey found that women aged 43-49 who had used oral contraceptives in the past had a significantly lower prevalence of severe nuclear sclerosis than women who had never used oral contraceptives, but there was no significant association in older age groups between past use of oral contraception and nuclear sclerosis. ${ }^{3}$ The Blue Mountains Eye study found no association between prevalence of cataract and past use of oral contraception in a population survey of women aged 49 and development of diabetic retinopathy in women over. ${ }^{4}$ Given the sparsity of the epidemiological evidence available, we have undertaken an analysis of the data on eye disease in the two large British cohort studies of the benefits and risks of oral contraception-namely, the Royal College of General Practitioners' (RCGP) Oral Contraception Study $^{5}$ and the Oxford-Family Planning Association (Oxford-FPA) contraceptive study. ${ }^{6}$ We summarise our findings here.

Material and methods

ROYAL COLLEGE OF GENERAL PRACTITIONERS' ORAL CONTRACEPTION STUDY

During a 14 month period beginning in May 1968, 1400 British general practitioners recruited 23000 women using oral contraceptives and a similar number who had never done so. ${ }^{5}$ The two groups were of similar age and all subjects were married or living as married. Most (98\%) were white. Information collected at recruitment included smoking habits, parity, social class, and previous medical history. At regular intervals since recruitment the general practitioners have supplied for each woman still under observation details of any hormonal preparations prescribed, all newly presenting episodes of illness and surgery, and, when appropriate, date and cause of death.

During the course of the study, three oral contraceptive user groups have evolvedcurrent users, former users, and never users. For each calendar month in which a woman uses the pill, one month is added to the period of exposure of current users. If that woman stops the pill, her subsequent periods of observation are included in the former user group unless she restarts, in which case she again contributes from the date of change to the current user periods of observation. If a woman who has never used oral contraceptives at recruitment subsequently starts to use the pill, her experience thereafter is allocated to the appropriate user groups. About one quarter of the original cohort currently remain under observation, most women being lost because they moved from the practice area of the recruiting doctor; all information is used, however, up to the time of loss.

The results relate to first ever episodes of a number of eye diseases occurring both in the community and in hospital, during the study using data available at November 1996. Cases diagnosed before recruitment or during pregnancy were excluded, together with the associated periods of observation. The incidence rates were directly standardised for age at diagnosis using the total RCGP study population for reference purposes (with the exception of 
Table 1 Rates of different eye diseases in the RCGP study and the Oxford-FPA study

\begin{tabular}{lclc}
\hline Condition & $\begin{array}{l}\text { RCGP Study } \\
\text { (No of cases) }\end{array}$ & $\begin{array}{l}\text { Oxford-FPA Study } \\
\text { (No of cases) }\end{array}$ & $\begin{array}{l}\text { Rate ratio } \\
\text { RCGP:Oxford-FPA }\end{array}$ \\
\hline Conjunctivitis & $100.0(5646)$ & $5.9(183)$ & 16.9 \\
Keratitis & $3.5(199)$ & $1.7(54)$ & 2.0 \\
Iritis & $3.4(187)$ & $1.6(51)$ & 2.1 \\
Lacrimal disease & $4.4(246)$ & $2.0(63)$ & 2.2 \\
Strabismus & $1.0(59)$ & $1.1(33)$ & 0.9 \\
Cataract & $2.9(170)$ & $1.8(48)$ & 1.6 \\
Glaucoma & $2.2(127)$ & $2.1(58)$ & 1.0 \\
Retinal detachment & $0.7(38)$ & $1.0(30)$ & 0.7 \\
Retinal vascular lesions & $1.2(71)$ & $0.9(25)$ & 1.4 \\
\hline
\end{tabular}

Rates are per 10000 person years of observation and are directly standardised for age ( 5 year age groups) using the combined study populations as the reference standard.

Table 1 where the combined populations of both the RCGP and the Oxford-FPA study were used as the reference standard). $95 \%$ confidence intervals were calculated on the assumption that the standard deviation of the $\log$ relative risk is equal to the sum of the reciprocals of the observed number of cases in the two groups being compared.

OXFORD-FAMILY PLANNING ASSOCIATION CONTRACEPTIVE STUDY

Between 1968 and 1974, 17032 white married women, aged 25-39, were recruited at 17 family planning clinics in different parts of England and Scotland. ${ }^{6}$ At recruitment, 9654 $(56 \%)$ of women were taking oral contraceptives, $4216(25 \%)$ were using a diaphragm, and $3162(19 \%)$ were using an intrauterine device. The women were followed up at the clinics or by post, telephone, or home visits. Information collected from each woman during follow up included details of any pregnancies and their outcome, changes in contraceptive practices, and reasons for referral to hospital either as an outpatient or an inpatient. Diagnoses on discharge from hospital were confirmed by obtaining copies of discharge letters, summaries, and pathology reports.

Of the 17032 women in the study, 15292 (90\%) remained under observation on reaching the age of 45 . At that age, each woman was allocated to one of three groups: (a) oral contraceptives never used, (b) oral contraceptives used for a total of 8 years or more, and (c) other durations of oral contraceptive use. Only the women in the first two groups (total 9401) were followed up from then on in the detailed way described above. Accordingly, women in group (c) have been omitted from the present analysis from the age of 45 onwards.

The analysis is based on data collected up to July 1994. Person years of observation were computed in the various groups of interest with the calculation of indirectly standardised rates (considering age at diagnosis) by the method described by Vessey et $a l^{6}$ (with the exception of Table 1 where a direct method was used). $95 \%$ confidence intervals around point estimates of relative risk are based on methods described by Breslow and Day. ${ }^{7}$ Analyses were conducted separately for women aged up to 45 and for women aged 45 or more. The results in the two sets of analyses were similar; accordingly, only the overall figures are given here.

\section{EYE CONDITIONS EXAMINED}

The conditions examined were: conjunctivitis (International Classification of Diseases, 8th Revision (ICD8), code 360); keratitis (ICD8 code 363); iritis (ICD8 code 364); lacrimal disease (ICD8 code 368); strabismus (ICD8 code 373); cataract (ICD8 code 374); glaucoma (ICD8 code 375); retinal detachment (ICD8 code 376); vascular lesions of retina (ICD8 code 377.0), excluding diabetic retinopathy (ICD8

Table 2 Relative risks for the various eye conditions in the RCGP study and the Oxford-FPA study according to oral contraceptive use. Never users of oral contraceptives have been taken as the reference category. Adjusted for age (5 year age groups)

\begin{tabular}{|c|c|c|c|c|c|}
\hline \multirow[b]{2}{*}{ Condition } & \multirow[b]{2}{*}{ Study } & \multirow[b]{2}{*}{ No cases } & \multicolumn{3}{|c|}{ Oral contraceptives } \\
\hline & & & Ever used & Recently used & Used in past \\
\hline \multirow[t]{2}{*}{ Conjunctivitis } & RCGP & 5646 & $1.1(1.0-1.1)^{\star}$ & $1.2(1.1-1.3)^{\star}$ & $1.0(1.0-1.1)$ \\
\hline & Ox-FPA & 183 & $1.3(0.9-1.9)$ & $1.7(1.3-2.2)^{\star}$ & $1.1(0.7-1.7)$ \\
\hline \multirow[t]{2}{*}{ Keratitis } & RCGP & 199 & $0.8(0.6-1.1)$ & $0.9(0.6-1.4)$ & $0.8(0.6-1.1)$ \\
\hline & Ox-FPA & 54 & $1.0(0.5-1.7)$ & $1.4(0.7-2.6)$ & $0.7(0.3-1.4)$ \\
\hline \multirow[t]{2}{*}{ Iritis } & RCGP & 187 & $0.8(0.6-1.0)$ & $0.7(0.5-1.0)^{\star}$ & $0.6(0.5-0.9)^{\star}$ \\
\hline & Ox-FPA & 51 & $1.1(0.6-2.0)$ & $1.1(0.5-2.4)$ & $1.1(0.6-2.1)$ \\
\hline \multirow[t]{2}{*}{ Lacrimal disease } & RCGP & 246 & $1.2(1.0-1.6)^{\star}$ & $1.4(1.0-1.9)^{\star}$ & $1.0(0.8-1.4)$ \\
\hline & Ox-FPA & 63 & $0.8(0.5-1.4)$ & $0.8(0.3-1.6)$ & $0.9(0.5-1.5)$ \\
\hline \multirow[t]{2}{*}{ Strabismus } & RCGP & 59 & $0.9(0.6-1.6)$ & $0.6(0.3-1.3)$ & $1.0(0.6-1.7)$ \\
\hline & Ox-FPA & 33 & $0.6(0.3-1.3)$ & $0.7(0.3-1.7)$ & $0.5(0.2-1.2)$ \\
\hline \multirow[t]{2}{*}{ Cataract } & RCGP & 170 & $0.8(0.6-1.0)$ & $0.3(0.2-0.6)^{\star}$ & $0.8(0.6-1.1)$ \\
\hline & Ox-FPA & 48 & $1.6(0.8-2.9)$ & $3.7(1.6-8.2)^{\star}$ & $1.1(0.6-2.3)$ \\
\hline \multirow[t]{2}{*}{ Glaucoma } & RCGP & 127 & $1.0(0.7-1.4)$ & $0.8(0.4-1.9)$ & $1.0(0.7-1.5)$ \\
\hline & Ox-FPA & 58 & $1.6(0.9-2.0)$ & $1.2(0.3-3.5)$ & $1.7(1.0-3.0)$ \\
\hline \multirow[t]{2}{*}{ Retinal detachment } & RCGP & 38 & $0.8(0.4-1.4)$ & $0.6(0.3-1.4)$ & $0.6(0.3-1.3)$ \\
\hline & Ox-FPA & 30 & $2.3(1.0-5.7)^{\star}$ & $2.1(0.6-7.0)$ & $2.4(1.0-6.1)$ \\
\hline \multirow[t]{2}{*}{ Retinal vascular lesions } & RCGP & 71 & $1.3(0.8-2.0)$ & $2.0(1.0-3.8)^{\star}$ & $1.0(0.6-1.7)$ \\
\hline & Ox-FPA & 25 & $1.9(0.8-4.7)$ & $2.4(0.4-9.2)$ & $1.9(0.7-4.6)$ \\
\hline
\end{tabular}

${ }^{\star} \mathrm{p}<0.05$.

Periods of observation (person years):

RCGP:

Never used 218974

Ever used 319652

Recently used 131090

Used in past 188562

Oxford-FPA:

Never used 123082

Ever used 185927

Recently used 73713

Used in past 112214 . 
Table 3 Retinal vascular lesions in the RCGP study and the Oxford-FPA study according to diagnosis. The data are numbers (\%) of patients

\begin{tabular}{lcc}
\hline Diagnosis & RCGP Study & Oxford-FPA Study \\
\hline Retinal vascular occlusion (arterial and unspecified) & $10(14.1)$ & $3(12.0)$ \\
Retinal vein thrombosis & $17(24.0)$ & $8(32.0)$ \\
Retinal haemorrhage & $16(22.5)$ & $8(32.0)$ \\
Vitreous haemorrhage & $16(22.5)$ & $3(12.0)$ \\
Central serous chorioretinopathy & $4(5.6)$ & $1(4.0)$ \\
Other and unspecified lesions & $8(11.3)$ & $2(8.0)$ \\
Total & $71(100.0)$ & $25(100.0)$ \\
\hline
\end{tabular}

code 250). The analysis was limited to first episodes of disease newly presenting during follow up. Some women had more than one diagnosis, so when all eye conditions were combined, the total number of events was smaller than the sum of the events for each problem examined separately. Each event was categorised according to the woman's contraceptive status at the time of the event. In both studies, events in current users were combined with those occurring within 12 months of discontinuation of oral contraceptives to produce a "recent users" category. It should be noted that this is a routine practice in the OxfordFPA study; it is implemented to minimise the distorting effects which might otherwise occur if women presenting with disease in general practice are taken off the pill some time before referral to hospital.

\section{Results}

Preliminary analyses of the data for the different eye conditions examining the effects of age, parity, social class, and smoking habits showed age to be the only factor which had an important and consistent effect on risk. Accordingly, the results presented for the oral contraceptive comparisons are adjusted for the effects of age alone.

Table 1 gives the numbers of cases and the age standardised rates for the different eye conditions in the two studies. It should be borne in mind that the RCGP data include diagnoses managed entirely in primary care as well as eye problems referred to hospital. The Oxford-FPA data on the other hand include only those eye problems that are referred to hospital. Not surprisingly, conjunctivitis emerges as a condition which is managed almost entirely in general practice. For many of the other diseases, the rates in the RCGP study are around twice those in the Oxford-FPA study; presumably this reflects the fact that not all patients with the eye diseases listed are referred to hospital. For strabismus, glaucoma, and retinal detachment, however, there is little difference between the rates in the two studies. Hospital referral would be expected in the great majority of patients presenting with these conditions.

Table 2 presents the findings for the various conditions in the two studies according to oral contraceptive use. The data shown are relative risks with $95 \%$ confidence intervals taking those never using oral contraceptives as the reference category. The results for conjunctivitis are consistent with a slight increase in risk in recent users of oral contraceptives, but the effect is small. The suggestion of a reduced risk of iritis in pill users in the RCGP study is not supported by the findings in the Oxford-FPA study. Likewise, the slightly increased risk of lacrimal disease in recent users of oral contraceptives in the RCGP study is balanced by a slightly reduced risk in the Oxford-FPA study. The results of the two studies for cataract are, however, markedly discrepant. Thus the RCGP study yields a low relative risk of 0.3 $(0.2-0.6)$ for this condition in recent users of oral contraceptives while the corresponding relative risk in the Oxford-FPA study is markedly elevated at $3.7(1.6-8.2)$. It should be noted, however, that this latter figure is based on 10 exposed cases and that only three of these cases underwent cataract surgery. Limiting the analysis to women undergoing cataract surgery (28 in all) yields a relative risk of $2.4(0.4-8.6)$ for recent users of oral contraceptives in the Oxford-FPA study. Further examination of the data on cataract in the two studies did not provide any additional clues as to the explanation for the difference in the results. Only 68 women in total in the two studies were reported to have suffered retinal detachment. The suggestion of an increased risk of this condition in pill users in the Oxford-FPA study was not supported by the findings in the RCGP study.

Retinal vascular lesions were more common in recent oral contraceptive users than in never users in the RCGP study, a difference which just reached statistical significance (relative risk $2.0(1.0-3.8)$ ). There was a similar increase in risk in the Oxford-FPA study which did not, however, approach statistical significance (relative risk $2.4(0.4-9.2))$. None the less, in view of the extensive evidence that oral contraceptives are a cause of vascular events elsewhere in the body, ${ }^{8}$ these results are of particular interest and we subjected them to closer analysis.

Firstly, we reviewed the individual study records for each woman suffering a retinal vascular lesion and arrived at the results given in Table 3. Given the small numbers of subjects, the distributions of the diagnoses in the two studies are closely similar save that there is a greater proportion of vitreous haemorrhage diagnosed in the RCGP study. This may reflect the lower diagnostic precision that would be expected in eye problems managed entirely in primary care. Of the 71 women with retinal vascular disease in the RCGP study, 24 (34\%) had a previous diagnosis of hypertension and $12(17 \%)$ a previous diagnosis of diabetes mellitus (three women had both conditions). These diagnoses were recorded much less frequently in the Oxford-FPA study; thus only three women $(12 \%)$ had a previous diagnosis of hypertension and one (4\%) of diabetes mellitus. These differences presumably reflect the fact that the Oxford-FPA study records disease events only for women referred to hospital.

We examined the association between oral contraceptive use and the risk of these specific retinal vascular diagnoses, but found that the increase observed in Table 2 was not concentrated in any one diagnostic group identified in Table 3. Indeed, for none of the individual diagnoses was the relative risk associated with 
oral contraceptive use significantly greater than one. Of course, numbers of cases in each category were small, so the confidence intervals were wide.

\section{Discussion}

The RCGP and the Oxford-FPA studies have accumulated over 850000 person years of observation in 63000 women, including 205000 person years in current/recent users and 301000 person years in past users. It is reassuring that no consistent evidence of any major eye problems has emerged from examination of these data sets which puts into context the earlier case reports which suggested that there might be links between use of oral contraception and eye disorders.

COMPARISON OF RESULTS IN THE TWO DATA SETS The two data sets are by and large consistent with each other. Both observed a small, but significant, increase in risk in conjunctivitis in recent oral contraceptive pill users. It is possible that this increase in risk is related, at least in part, to the difficulties which some oral contraceptive users experience with contact lenses. ${ }^{9}$ A significant increase in risk of lacrimal disease and a significant reduction in risk of iritis were observed in the RCGP study, but these effects were not confirmed in the Oxford-FPA study. Nevertheless, there is considerable overlapping of the confidence intervals for the estimates of relative risk for these two conditions in the studies, so that results are in keeping with each other. Similarly, a significant excess of risk of retinal detachment in ever users of oral contraception in the Oxford-FPA study was not observed in the RCGP study, but again, the confidence intervals overlapped. The area where there was the most consistent evidence of an excess risk was retinal vascular disease. Both studies estimated the relative risk as being two or above, statistically significant in the RCGP study, not so in the Oxford-FPA study. Closer examination of the diagnoses did not, however, reveal that the excess risk was confined to a specific diagnosis.

The one diagnosis where there was a significant discrepancy was in cataract, where the RCGP study found a significant reduction in risk in oral contraceptive users, while the Oxford-FPA study found the opposite. It is difficult to explain this discrepancy, but the reason possibly lies in ascertainment bias which is considered below.

POSSIBLE BIASES

There are three important biases that need to be considered in interpreting the results of these studies: selection bias, ascertainment bias, and bias arising from differential loss to follow up.

Selection bias is possible since women with risk factors such as hypertension, diabetes, or hyperlipidaemia may have been encouraged by their general practitioners to use alternative methods of contraception. Therefore, women who were prescribed oral contraception were probably at lower risk of retinal vascular lesions than women who were not. This will have reduced any apparent risk of retinal vascular lesions in users of oral contraception. Therefore, the excess relative risk of these diagnoses in these data sets are likely to be an underestimate rather than an overestimate.

Ascertainment bias might occur if doctors had a lower threshold for considering ophthalmic diagnoses in women taking oral contraception. This is possible, since at the time the cohorts were being recruited, a number of case reports were being published linking oral contraception to various eye diseases. For example, in one review of the ophthalmic complications of oral contraception, the author emphasised the "necessity of ophthalmologic examinations during contraceptive regime". ${ }^{10}$ Such diagnostic vigilance is likely to have been greater in family planning clinics than in general practice, so may explain why the estimates of relative risk were higher in the Oxford-FPA study than in the RCGP study for users of oral contraception for every diagnosis except lacrimal disease. Such bias may have contributed to the differences that were observed with regard to risk of cataract in the two studies. Ascertainment bias will have resulted in an overestimate of risk of eye disease in oral contraceptive users, so will have counterbalanced the effects of selection bias, though it is impossible to say which of these two biases will have had a greater effect.

Loss to follow up is an unlikely source of important bias in these studies. Loss to follow up in the Oxford-FPA study was low. The RCGP study experienced greater loss to follow up, mostly because of women leaving the practice area. However, the characteristics of pill users lost to follow up are similar to those of non-users lost to follow up. ${ }^{11}$ Furthermore, a recent analysis of mortality data from the RCGP study failed to reveal any important bias resulting from the high rate of attrition. ${ }^{12}$

\section{Conclusion}

With the exception of retinal vascular disease, there is no consistent evidence of important increases in risk of eye diseases in users of oral contraception. It has been useful to consider the results of these two data sets in combination, since taken on their own, each suggested links to specific eye diseases which were not confirmed by the other study.

Royal College of General Practitioners' Study: We thank the 1400 general practitioners who contributed data to the RCGP Oral general practitioners who contributed data to the RCGP Oral
Contraception Study. This study currently receives support from the Royal College of General Practitioners, Schering AG, Schering Health Care Ltd, and Wyeth Ayerst International Inc. Oxford Family Planning Association Study: We thank Mrs D Collinge, Mrs J Winfield, and the research assistants, doctors, and nurses who worked in the participating clinics for their important contribution. We are also grateful to the Medical Research Council and the Knott Family Trust for financial support.

1 Wood JR. Ocular complications of oral contraceptives. Ophthalmic Sem 1977;2:371-402.

2 Garg SK, Chase P, Marshall G, et al. Oral contraceptives and renal and retinal complications in young women with insulin dependent diabetes mellitus. $\mathscr{A} A M A$ 1994;271: insulin dep9-102.

3 Klein BEK, Klein R, Ritter LL. Is there evidence of an estrogen effect on age related lens opacities? The Beaver Dam Eye Study. Arch Ophthalmol 1994;112:85-91. 
4 Cumming RG, Mitchell P. Hormone replacement therapy, reproductive factors, and cataract. The Blue Mountains Eye Study. Am f Epidemiol 1997;145:242-9.

5 Royal College of General Practitioners. Oral contraceptives and health. London: Pitman Medical, 1974.

6 Vessey MP, Doll R, Peto R, et al. A long-term follow-up study of women using different methods of contraception - an interim report. F Biosoc Sci 1976;8:373-427.

7 Breslow NE, Day NE. Statistical methods in cancer research Vol II. The design and analysis of cohort studies. Lyons: IARC, 1987.

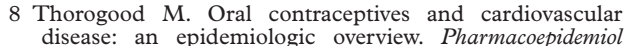
Drug Safety 1993;2:3-16.
9 Radnot M, Follmann P. Ocular side-effects of oral contraceptives. Ann Clin Res 1973;5:197-204.

10 Varga $M$. Recent experiences on the ophthalmologic complications of oral contraceptives. Ann Ophthalmol 1976;8:925-34

11 Hannaford PC, Kay CR, Vessey MP, et al. Combined oral contraceptives and liver disease. Contraception 1997;55: 145-51.

12 Beral V, Hermon C, Kay C, et al. Mortality in relation to method of follow-up in the Royal College of General Practioners' Oral Contraception Study. In: Hannaford PC, Webb AMC, eds. Evidence-guided prescribing of the pill. Parthenon: Carnforth, 1996:327-39. 